\title{
Cognitive Behavioral Therapy in Treating Veterans with PTSD: Efficiency and Multiculturalism
}

\section{Nadia L. Williams, LCSW}

School of Social Work, Fayetteville State University, 1200 Murchison Road, Fayetteville, NC 28301, United States.

Article Details
Article Type: Research Article
Received date: $15^{\text {th }}$ December, 2020
Accepted date: $22^{\text {nd }}$ January, 2021
Published date: $25^{\text {th }}$ January, 2021
"Corresponding Author: Nadia L. Williams, LCSW, School of Social Work, Fayetteville State University, 1200 Murchison
Road, Fayetteville, NC 28301, United States. E-mail: nwilli29@uncfsu.edu
Citation: Williams, N.L. (2021). Cognitive Behavioral Therapyin Treating Veterans with PTSD: Efficiency and Multicultur-
alism. J Ment Health Soc Behav 3(1):129. https://doi.org/10.33790/jmhsb1100129
Copyright: C2021, This is an open-access article distributed under the terms of the Creative Commons Attribution License
4.0, which permits unrestricted use, distribution, and reproduction in any medium, provided the original author and source are
credited.

\section{Abstract}

This paper discusses cognitive-behavioral therapy and its implications in treating veterans with PTSD. This paper reviews current literature and research on the adaption of CBT for varying cultural factors and the efficiency of CBT in the treatment of PTSD. Research shows that CBT is effective in the treatment of PTSD and continues to be a first-line treatment intervention. CBT has been adapted for varying diagnosis and symptomology and continues to evolve. Future implications and research are needed on how varying elements of CBT are effective with veterans.

Keywords: Cognitive-behavioral therapy, Evidenced-based treatments, Veterans, Post-traumatic stress disorder, Anxiety disorders, Depression, Multiculturalism

\section{Introduction}

\section{Description of Practice Method}

Hofmann et al. [1] discussed the theoretical background of cognitive-behavioral therapy (CBT). The authors stated that CBT is a part of a class of interventions where the basic premise is that mental disorders and psychological distress are affected by cognitive factors. The basis for this treatment is that maladaptive thoughts contribute to the maintenance of emotional distress and behavioral problems. The maladaptive thoughts that can be affected include beliefs about the world, the self and the future, that exacerbate thoughts, both specific and automatic thoughts as it relates to certain situations. These types of cognitive treatments assist in the change of negative thoughts that lead to emotional distress and problematic behaviors.

Hofmann et al. [1] continue in discussing the overall goals of treatment being symptom reduction, improvement in overall functioning, and placing the disorder and symptoms into remission. The patient achieves this goal by being an active collaborator in the problem-solving process. For the patient, the problem-solving process involves testing and challenging the validity of negative thoughts and to change negative behaviors. CBT is concerned with a group of related interventions that combine cognitive, behavioral, and emotion-focused techniques. CBT is also concerned with how cognitive factors, physiological, emotional, and behavioral elements impact the disorder.

González-Prendes et al. [2] found several basic assumptions about $\mathrm{CBT}$ :The basic assumptions for $\mathrm{CBT}$ are that cognitive processes and content are accessible and can be known; our thoughts and beliefs mediate the way we process information and consequently affect our emotional and behavioral responses; and maladaptive cognitions can be intentionally targeted and changed in a more rational and realistic direction, thus relieving symptoms and increasing functionality. (p. 21)

\section{Methods, Central Techniques and Strategies of CBT}

Nathan and Gorman et al. [3] describe the methods and techniques of CBT. CBT is considered a short-term, directive therapy designed to change the patient's negative view of the self, world, and future. The treatment consists of 16-20 sessions over the course of approximately 12-16 weeks. The treatment starts with information on the treatment, the goals, and the rationale underlying the treatment. In early sessions the goals are to implement strategies to increase behavioral activities. Around week 3, self-monitoring techniques are introduced with a goal of examining the relationship between thoughts and feelings. Patients are taught to examine their thoughts for "logical errors that include arbitrary inference, selective abstraction, overgeneralization, magnification and minimization, personalization, and dichotomous thinking" (p. 386). In the middle of treatment, around session 8 or 9 the idea of beliefs underlying negative and positive thoughts are introduced. The goal of treatment at this stage is to focus on changing negative thoughts. At the end of treatment around sessions 14 to 16 , the goal of treatment focuses on termination and the use of cognitivebehavioral strategies to reduce relapse.

Burgin et al. [4] indicated that mental health professionals working with veterans and their families need to be empathetic and understanding of the unique qualities and experiences their clients present in order to provide competent care. Those working with service members have a responsibility to not only understand who their clients are culturally and, in the military, but also how their experiences impact who they are outside of the service.

CBT can be and should be adapted for the client being served, to include multicultural sensitivity and awareness. Farrand et al. [5] conducted a qualitative study on adapting CBT to treat depression for Armed Forces (AF) veterans. They found that adapting CBT to incorporate the $\mathrm{AF}$ specific culture of language, metaphors and analogies used was helpful in engagement in CBT with veterans.

In addition to adapting CBT, clinicians should also take time to be culturally competent in their client's symptomology and insight into their problems and symptoms. For instance, [6] discuss various cultural conditions and symptoms. An example of a culture-bound syndrome is Ataque de Nervios and can be translated as attack of the nerves. The symptoms of the syndrome can be described as trembling, disassociation, and crying. In Latinx culture, this could be used to describe situational occurrences such as a traumatic event, or for others an ongoing occurrence for someone who has no situational trigger. In a western worldview, this might be seen as anxiety or a panic attack. Being culturally sensitive and aware as a clinician utilizing interventions like CBT is imperative in terms of engaging the client in treatment as realizing effective treatment outcomes. Understanding your client's viewpoint, culture and experiences 
whether they be military culture, or race and ethnicity directly effects treatment outcomes.

\section{Clients Best Served by CBT}

The field practicum site examined in relation to the treatment model CBT is the Durham Veterans Affairs Healthcare System (DVAHCS) Post-Traumatic Stress Disorder (PTSD) Clinic. At the DVAHCS the primary population served is veterans. Within the PTSD clinic the primary population served is combat veterans with PTSD. With Iraq and Afghanistan conflicts ending and service personnel having a higher prevalence of PTSD, the need to treat veterans is a major goal of the DVAHCS.

\section{Practice Method Fit}

Cukor et al. [7] state that the diagnostic criteria for PTSD was first introduced to the DSM-III in the 1980's and that many clinical trials have been conducted to determine how to ameliorate the symptoms of the PTSD. Several methods have been examined and found to be effective in treating PTSD including pharmacological approaches that directly affect the symptoms, and CBT which treats related symptoms. "The efficacy of CBT trauma-focused treatments including prolonged exposure, cognitive processing therapy (CPT) and eye movement desensitization and reprocessing (EMDR) have received support"'(p. 2).

\section{Research on the Effectiveness of CBT}

Margolies et al. [8] discussed the impact of sleep disturbances in PTSD and how this can reduce or exacerbate the severity of PTSD. The authors found that sleep disturbances, including insomnia and nightmares, have been found to be predictive in the development of PTSD from one month to one year later. The use of CBT for the treatment of sleep disorders involves several components including sleep restriction, the control of stimuli, sleep hygiene, and thought restructuring all of which have been effective approaches to treating PTSD that is comorbid with insomnia. The authors found evidence for clinicians treating PTSD to also assess sleep disturbances and treating the comorbid disorders with CBT.

Tolin et al. [9] indicated that CBT is effective in treating many different psychiatric disorders. The author holds that CBT performs better than other forms of treatment at the conclusion of treatment. Individuals that receive CBT show lessened symptom severity after treatment than patients receiving other therapies. The effectiveness of CBT is even more evident at six months and one-year post-treatment. These time frames imply that CBT is effective and reliable across time. The author suggests the use of CBT as a first-line psychosocial treatment for most patients.

Friedman [10] stated that CBT should be made the treatment of choice for all individuals diagnosed with PTSD. The reason CBT has been found to be effective is because it addresses conditioned fear and cognitive distortions that are associated with PTSD. Hofmann et al. [1] conducted an analysis of several studies that compared the response rates of $\mathrm{CBT}$ and other treatments. In those studies, $\mathrm{CBT}$ was found to have a higher response rate than other treatments.

González-Prendes and Brisebois [2] indicated that extensive research supported the effectiveness of CBT for many psychosocial issues. CBT has been noted as one of the most widely researched and published models of treatment. The authors stated that more than 325 studies were published on CBT outcomes and they indicated the efficacy of the treatment model. Due to CBT being widely researched and proven to be effective, it has been the popular choice of treatment for practitioners utilizing evidence-based practices.

CBT is used and adapted often to treat specific symptoms and conditions in veterans, and civilians with improved outcomes. Brown et al. [11] provided an example of the use of CBT in treating 902 patients for depression and observed that at the conclusion of the treatment $47 \%$ reported no suicidal ideation (SI), $45 \%$ had SI but no intent for suicide, and $64 \%$ showed a decrease in the odds of SI. Suicide and depression are both symptoms and risks for veterans and others with PTSD.
David et al. [12] name CBT as the gold standard for first line psychotherapies for many diagnoses, including depression and anxiety. Cuijpers et al. [13] reported that approximately $54 \%$ of the trials for depression, and approximately $20 \%$ of the trials for anxiety were comparable to pill placebo or treatment as usual comparisons. They continue by describing CBT as the gold standard because it is the most researched psychological intervention. There have not been other psychological interventions that perform better empirically than CBT. CBT has consistently adapted to the changes in the cognition and behavior over time. It is reliable.

Carpenter et al. [14] conducted a meta-analysis on the effectiveness of CBT on several anxiety disorders to includea cute stress disorder, generalized anxiety disorder (GAD), obsessive-compulsive disorder (OCD), panic disorder (PD), PTSD or seasonal affective disorder (SAD). The review included 41 trials which was an increase of $59 \%$. The patients were randomly assigned to CBT or placebo for the treatment of the identified anxiety disorders. They found that post-treatment and follow up resulted in improvement in anxiety, depression and quality of life, and better treatment responses overall. These results also indicate that CBT is effective in improving symptomology for various anxiety disorders, and for improving the client's overall life.

Kaczkurkin and Foa [15] concluded that of the cognitive behavioral therapies, exposure and cognitive methods both are highly used and are both highly effective in the treatment of anxiety disorders including PTSD. The authors also point to the need for more extensive research on which components are most effective for the varying conditions as there can be overlap in methods used.

Review of research on the efficiency of CBT shows that CBT continues to be a first-line treatment for various conditions, specifically anxiety disorders which includes PTSD. CBT has shown to be effective in studies over the last several decades. CBT is effective at improving not only client symptomology, but the client's whole life and engagement in treatment. CBT has adapted over the course of its use as a psychological treatment, but CBT still has room for improvement. Further exploration and clarification as to what portions are most effective for various populations and diagnosis are needed, as well as exploring how CBT can be adapted for various cultural groups.

\section{National Association of Social Workers (NASW) Code of Ethics Critical Assessment Self-Determination}

González-Prendes and Brisebois [2] discussed CBT as it relates to the NASW Code of Ethics. In the area of self-determination, the authors found that respect for the worth of the person is one of the primary principles of CBT. Clinicians using CBT accept their clients for who they are and meet them where they are regardless of how the client feels or behaves. CBT does not use labels or make judgements about the individuals utilizing the treatment, and places value on broadmindedness. In CBT judgment, stereotyping, and using derogatory terms are seen as negative thoughts, and the goal $\mathrm{CBT}$ is to retrain negative thoughts.

Rather than make judge ments about the individual, CBT enables clinicians to judge how negative thoughts and behaviors effect the individual and their goals. Therapists using this model train their clients to accept themselves fully regardless of their flaws, and this is important in having self-worth which helps to repair the patients functioning. The author continues by stating that using problemsolving within the treatment model of CBT encourages self-efficacy and client self-determination.

Kaczkurkin and Foa [15] explain that the basis of CBT is to change maladaptive emotional responses through a change in behaviors and/ or thoughts. By virtue of an individual changing behaviors, thoughts, and emotions, self-determination enables change for the better. CBT helps veterans to identify areas they want to improve, the behaviors and thoughts that are identified help them to develop a plan to change for behavioral change. 


\section{Empowerment}

González-Prendes and Brisebois [2] indicated that in CBT client empowermentis the recognition of the ability of clients to engage in self-awareness. In social work, clients are considered the experts in their lives and the issues that plague them. Emphasizing this ensures client empowerment in the treatment process. Another point is that clients have the ability to change their thoughts and beliefs and this empowers clients to develop healthier emotional and behavioral responses to their life situations. The final point the authors made was the importance of assisting clients in developing skills, both cognitive and behavioral, and to prepare them for various life events when the treatment is finished, and the clinician is no longer involved.

Lewis-Fernandez and Kirmayer [6] suggest that exploring with clients what their current coping skills are, and how they perceive their problems makes the client the expert in his or her own life thus empowering them to engage in treatment. When clinicians working with veterans allow them to lead their interventions and treatments based on their experiences and resilience, this empowers clients to seek change and to be an active participant in the change process. This is evidence of CBT's effectiveness in engaging veterans but also is evidence of how empowerment positively impacts intervention efficacy. Further, they give an example of using the language of addiction when describing gamers. For some gamers, their interest in the activity is positive and allows them to have an enjoyable outlet. On the other hand, some gamers use the activity as a way to avoid life and it becomes a negative coping mechanism. If clinicians only view their client's problems from their viewpoint, the way they carry out CBT could be ineffective. Clinicians need to have an understanding of how their client perceives their problems and this comes from empowering clients to lead and engage in their treatment.

\section{Culturally Sensitive Practice}

González-Prendes and Brisebois [2] continue by pursuing a discussion of culturally sensitive practice indicating that CBT has primarily been researched from a Eurocentric viewpoint and that more research is needed in the area of multiculturalism. The authors continued by stating that although there is a need for more culturally sensitive practice in the CBT model, this is a weakness of many treatment models, and the field in general.

Farrand et al. [5] discusses the need for inclusive language when adapting CBT for different groups, particularly AF Veterans. This speaks to the need for CBT to be inclusive and culturally sensitive. When clinicians use culturally sensitive practices in CBT treatment, they will improve client outcomes, but also ensure that they are adhering to the NASW Code of Ethics which guides practice with the client and practices of the profession.

\section{Conclusion}

In the treatment of PTSD in veterans, CBT has been found to be the most effective treatment in many disorders including PTSD which is also described as an anxiety disorder. Due to outcome studies demonstrating the effectivenessof CBT, it is imperative for clinicians to examine their clients' needs and determine which treatment is most effective for them and their unique situations. Research has shown that CBT can be adapted for various symptoms, cultures, and symptom severities. Due to CBT being a broad treatment categorically, and the fact that several other treatments borrow from this model, it appears that CBT should be the first treatment assessed for fit with clients' needs.

Clinicians using CBT with clients should ensure that they are being culturally sensitive in their treatmentand should also ensure that they have examined all areas of the client's life that is impacted so they can be holistic in their treatment and approach. Since CBT is an evidencebased treatment model for many psychological disordersin cluding PTSD, it is highly utilized in the PTSD Clinic at the DVAHCS.

CBT involves the change in negative cognitive and behavioral actions and seeks to change the way the client views their life and beliefs. In treating PTSD in veterans, it is important to examine veteran's beliefs about the facts of the trauma and also how their thoughts and behaviors are impacted. By changing the individuals thought process and behaviors, this impacts their symptomology, and improves their overall well-being. CBT is most effective in giving clinicians and veterans an evidence-based framework for which they can work to improve the cognitions and/or behaviors and ultimately to improve their lives and belief systems.

Conflicts of interest/Competing interests: Authors report no conflict or competing interest.

\section{References}

1. Hofmann, S. G., Asnaani, A., Vonk, I. J., Sawyer, A. T., \& Fang, A. (2012). The efficacy of cognitive behavioral therapy: a review of meta-analyses. Cognitive therapy and research, 36(5), 427-440.http://dx.doi.org/10.1007/s10608-013-9595-3

2. González-Prendes, A. A., \& Brisebois, K. (2012). Cognitivebehavioural therapy and social work values: A critical analysis. Journal of Social Work Values and Ethics, 9(2), 2-21.

3. Nathan, P. E., \& Gorman, J. M. (Eds.). (2015). A guide to treatments that work. Oxford.

4. Burgin,E.E., Prosek, E.A., \&Atkins, K. M. (2017). Mental health and the U.S. Military: The need for counselor competencies. Journal of Military and Government Counseling.5(1), 2-19.

5. Farand, P., Mullan, E., Rayson, K., Englebrecht, A., Mead, K., \& Greenberg, N. (2019) Adapting CBT to treat depression in Armed Forces Veterans: Qualitative study. Behavioural and Cognitive Psychotherapy. 47(5), 530-540.https://doi. org/10.1017/S1352465819000171

6. Lewis-Fernandez, R., \& Kirmayer, L. J. (2019). Cultural concepts of distress and psychiatric disorders: Understanding symptom experience and expression in context. Transcultural Psychiatry. 56(4), 786-803.https://doi.org/10.1177\%2F1363461519861795

7. Cukor, J., Spitalnick, J., Difede, J., Rizzo, A., \& Rothbaum, B. O. (2009). Emerging treatments for PTSD. Clinical psychology review, 29(8), 715-726. https://doi.org/10.1016/j. cpr.2009.09.001

8. Margolies, S. O., Rybarczyk, B., Vrana, S. R., Leszczyszyn, D. J., \& Lynch, J. (2013). Efficacy of a cognitive-behavioral treatment for insomnia and nightmares in Afghanistan and Iraq veterans with PTSD. Journal of clinical psychology, 69(10), 1026-1042.

9. Tolin, D. F. (2010). Is cognitive-behavioral therapy more effective than other therapies? A meta-analytic review. Clinical psychology review, 30(6), 710-720. http://dx.doi.org/10.1016/j. cpr.2010.05.003

10. Friedman, M. J. (2006). Posttraumatic stress disorder among military returnees from Afghanistan and Iraq. American Journal of Psychiatry, 163(4), 586-593.

11. Brown, G. K., Bradley, K. E., Trockel, M., Gordienko, M., Yesavage, J., \& Taylor, B. (2016). Effectiveness of cognitive behavioral therapy for veterans with depression and suicidal ideation. Archives of Suicide Research, 20(4), 677-682.https:// doi.org/10.1080/13811118.2016.1162238

12. David, D., Cristea, I., \& Hofmann, S. G. (2018). Why cognitive behavioral therapy is the current gold standard of psychotherapy. Frontiers in Psychiatry. 9(4), 1-3.https://doi.org/10.3389/ fpsyt.2018.00004

13. Cuijpers, P., Cristea, I. A., Karyotaki, E., Reijnders, M., Huibers, M. J. H. (2016). How effective are cognitive behavior therapies for major depression and anxiety disorders? A metaanalytic update of the evidence. World Psychiatry. 15(3), 245258.https://doi.org/10.1002/wps.20346 
14. Carpenter, J. K., Andrews, L. A., Witcraft, S. M., Powers, M. B., Smits, J. A. J., \&Hofmann, S. G. (2018). Cognitive behavioral therapy for anxiety and related disorders: A metaanalysis of randomized placebo-controlled trials. Depression and Anxiety.35(6), 502-514.https://doi.org/10.1002/da.22728
15. Kaczkurkin, A. N., \& Foa, E. B. (2015). Cognitive-behavioral therapy for anxiety disorders: an update on the empirical evidence. Dialogues in Clinical Neuroscience. 17(3), 337-346. https://doi.org/10.31887/dens.2015.17.3/akaczkurkin 